\title{
Carriage of Class 1 integrons and molecular characterization of intI1 gene in multidrug-resistant Salmonella spp. isolates from broilers
}

\author{
Renu Gupta ${ }^{1}$, Sneh Lata Chauhan ${ }^{1}$, Sunil Kumar ${ }^{1}$, Naresh Jindal ${ }^{1}$, N. K. Mahajan ${ }^{1}$ and V. G. Joshi ${ }^{2}$ \\ 1. Department of Veterinary Public Health and Epidemiology, College of Veterinary Sciences, Lala Lajpat Rai University \\ of Veterinary and Animal Sciences, Hisar, Haryana, India; 2. Department of Animal Biotechnology, College of Veterinary \\ Sciences, Lala Lajpat Rai University of Veterinary and Animal Sciences, Hisar, Haryana, India. \\ Corresponding author: Renu Gupta, e-mail: renu.gupta@luvas.edu.in \\ Co-authors: SLC: chauhansnehlata90@gmail.com, SK: bishnoi.sunil88@gmail.com, NJ: nareshjindal1@gmail.com, \\ NKM: mahajan448@rediffmail.com, VG]: vinaygjoshi18@gmail.com \\ Received: 12-12-2018, Accepted: 12-03-2019, Published online: 25-04-2019
}

doi: 10.14202/vetworld.2019.609-613 How to cite this article: Gupta R, Chauhan SL, Kumar S, Jindal N, Mahajan NK, Joshi VG (2019) Carriage of Class 1 integrons and molecular characterization of intI1 gene in multidrug-resistant Salmonella spp. isolates from broilers, Veterinary World, 12(4): 609-613.

\begin{abstract}
Aim: The present study was conducted with the following aims: (i) To screen the Salmonella spp. isolates recovered from suspected cases of fowl typhoid for carriage of Class 1 integrons and analyze their association with antimicrobial resistance and (ii) to carry out molecular characterization and phylogenetic analysis of Class 1 integron-integrase (intI1) gene.
\end{abstract}

Materials and Methods: A total of 43 Salmonella isolates were subjected to polymerase chain reaction (PCR) assay to determine the presence of Class1 intI1. Differences between different serotypes in relation to their carriage of integrons and the differences between strains containing or not containing an integron and being resistant to different antimicrobials were analyzed by Fisher exact test using STATA ${ }^{\text {TM }}$ (StataCorp, College Station, TX). Phylogenetic analysis was carried out using MEGA6 software.

Results: Out of 43 isolates, $40(93.02 \%)$ were found positive for Class 1 integrons. 35/40 (87.5\%) intI1-positive isolates were multidrug resistance (MDR) (resistant to $\geq 4$ antibiotics), which support the hypothesis of an association between the presence of Class 1 integrons and emerging MDR in Salmonella. There was no significant difference among isolates resistant to different antimicrobials in Class 1 integron carrying isolates and the Class 1 integron negative isolates ( $\mathrm{p}<0.05)$. Further, there was no significant difference among different serotypes in respect of their carriage of Class 1 integrons.

Conclusion: It can be concluded that the high prevalence of Class 1 integrons indicates a high potential of Salmonella isolates for horizontal transmission of antimicrobial genes, especially among Gram-negative organisms.

Keywords: antimicrobial resistance, Class 1 integrons, phylogenetic analysis, Salmonella.

\section{Introduction}

Integron system is one of the important dynamic mechanisms in the evolution of multidrug resistance (MDR) which helps bacteria to acquire resistance genes in novel combinations enabling them to resist several antimicrobial agents and is frequently associated with the development of MDR in Gram-negative bacteria [1]. Integrons are capable of capturing and excising gene cassettes.

There are two types of integrons, chromosomal integrons and mobile integrons (MIs). MIs are of five classes - Class 1-5 [2]. Class 1 integrons, the most common type, mostly found as part of the Tn21 or Tn402 transposon family, have been detected in bacteria in many regions and have been identified as a primary source of antimicrobial resistance (AMR) genes and suspected to serve as reservoirs capable of exchanging

Copyright: Gupta, et al. Open Access. This article is distributed under the terms of the Creative Commons Attribution 4.0 International License (http://creativecommons.org/licenses/by/4.0/), which permits unrestricted use, distribution, and reproduction in any medium, provided you give appropriate credit to the original author(s) and the source, provide a link to the Creative Commons license, and indicate if changes were made. The Creative Commons Public Domain Dedication waiver (http://creativecommons.org/ publicdomain/zero/1.0/) applies to the data made available in this article, unless otherwise stated. resistance genes in a variety of Gram-negative bacteria $[3,4]$. They contain a $5^{\prime}$ conserved segment $\left(5^{\prime} \mathrm{CS}\right)$ and a $3^{\prime}$ conserved segment $\left(3^{\prime} \mathrm{CS}\right)[5]$.

The prevalence of integrons in Salmonella spp. of avian origin has not been previously reported from India. The present study was conducted with the following objectives: (i) To screen the Salmonella spp. isolates recovered from suspected cases of fowl typhoid for carriage of Class 1 integrons and analyze their association with AMR and (ii) to carry out molecular characterization and phylogenetic analysis of integron-integrase (intIl) gene.

\section{Materials and Methods}

\section{Ethical approval}

This study did not involve any live humans or animals and therefore no ethical approval was required.

\section{Isolates}

Sample collection, isolation, and biochemical characterization of Salmonella spp., serotyping, in vitro antimicrobial sensitivity patterns of the Salmonella isolates to various antimicrobials, and genomic DNA preparation has been described in our previous publication [6]. 
Screening of isolates for carriage of Class 1 integrons

Out of 45 flocks investigated for fowl typhoid, 43 Salmonella isolates were analyzed for the presence of Class 1 integrons according to the method previously described [7], with some modifications (annealing temperature of $56^{\circ} \mathrm{C}$ used instead of $54^{\circ} \mathrm{C}$ ), using primers intIl-F "ACGAGCGCAAGGTTTCGGT" and intI1-R "GAAAGGTCTGGTCATACATG."

\section{Statistical analysis}

A $95 \%$ confidence interval for carriage of integrons in different serotypes was calculated using STATA $^{\mathrm{TM}}$. Differences between different serotypes in relation to their carriage of integrons and the differences between strains containing or not containing an integron and being resistant to different antimicrobials were analyzed by Fisher exact test, using STATA ${ }^{\text {TM }}$ (StataCorp, College Station, TX).

\section{Sequencing of intI1 polymerase chain reaction (PCR) products}

Representative PCR products were got sequenced in the university facility using an ABI $3130 x \mathrm{~L}$ sequencer. The products were got accessioned in GenBank (accession no. MF346348.1, MF346759.1, MF346760.1, and MF346761.1).

\section{Phylogenetic analysis}

The related sequences (23) obtained during the BLAST search were retrieved from GenBank, and multiple sequence alignment was carried along with nucleotide sequences obtained from the present study. All the 27 sequences were aligned by ClustalW in MEGA6 software. The evolutionary history was inferred using the maximum likelihood method based on the Tamura 3-parameter model. Initial tree(s) for the heuristic search was obtained automatically by applying neighbor-join and BioNJ algorithms to a matrix of pairwise distances estimated using the maximum composite likelihood approach and then selecting the topology with superior log-likelihood value. Codon positions included were $1^{\text {st }}+2^{\text {nd }}+3^{\text {rd }}+$ Noncoding. All positions containing gaps and missing data were eliminated. There were a total of 290 positions in the final dataset. Evolutionary analyses were conducted in MEGA6 using 100 bootstrap replicates [8].

\section{Results}

Of the total of 43 Salmonella isolates subjected to PCR assay to determine Class1 integron integrase (intI1), $40(93.02 \%)$ were found positive for Class 1 integrons (Figure-1). 35/40 (87.5\%) intI1-positive isolates were MDR (resistant to $\geq 4$ antibiotics).

There was no significant difference among isolates resistant to different antimicrobials in Class 1 integron carrying isolates and the Class 1 integron negative isolates $(\mathrm{p}<0.05)$ (Table-1). Further, there was no significant difference among different serotypes in respect of their carriage of Class 1 integrons (Table-2).

Phylogenetic tree derived by analyzing and comparing the intIl gene sequences obtained from the current study and the published NCBI sequences is illustrated in Figure-1. Neighbor-joining phylogenetic tree indicated two distinct clads: First clad comprising all of the four isolates of the current study and almost all other isolates retrieved for analysis and the second clad comprising two isolates: Salmonella Brandenburg (NC 010500.1/0) from Spain and Salmonella Typhimurium (JQ345502.1) from Hungary (Figure-2).

\section{Discussion}

Of the total of 43 Salmonella isolates subjected to PCR assay to determine Class 1 integron integrase (intI1), 40 (93.02\%) were found positive for Class 1 integrons. 35/40 (87.5\%) intI1-positive isolates were MDR (resistant to $\geq 4$ antibiotics). The prevalence of integrons in Salmonella varies from country to country and depends on the origin of the isolates as discussed below.

There are different reports of the prevalence of Class 1 integrons in Salmonella isolates from different parts of the world. The Overall high positive rate of $66.5 \%$ of Class 1 integrons in multidrug-resistant Salmonella enterica serovar Indiana (87.2\%) and Enteritidis (50.8\%) isolated from chickens in Eastern China has been reported [9]. Class 1 integrons were present in 35 strains (38\%) of biotype gallinarum (90 strains) isolated from chickens in Korea [10]. The intI1 gene was present in $31 \%$ of the Salmonella isolates from broiler chickens, pigs, and meat products in Thailand and Cambodia [11]. The prevalence of Class 1 integrons in Salmonella isolates from humans and animals in Vietnam was found to be $28 \%$ [12], whereas, in China, Class 1 integrons were detected in 10/62 (16.13\%) Salmonella isolates from different retail foods [9]. A high prevalence of Class 1 integron in drug-resistant Salmonella enterica serovar Enteritidis isolates of poultry origin from Iran (17/30, $56.66 \%$ ) has also been reported [13].

In our study, there was no significant difference among isolates resistant to different antimicrobials

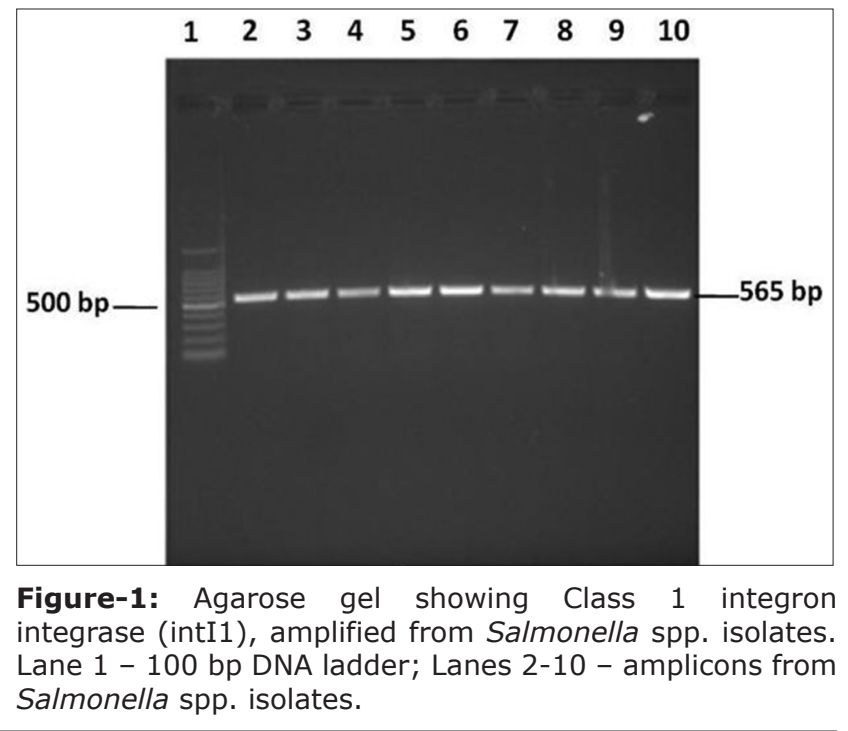


in Class 1 integron carrying isolates and the Class 1 integron negative isolates $(p<0.05)$. Further, there was no significant difference among different serotypes in respect of their carriage of Class 1 integrons.

In contrast, a significant relation was found for the presence of Class 1 integrons and resistance to trimethoprim, sulfonamides, and tetracycline in Escherichia coli isolated form laying hens in Belgium [7]. Similarly, Salmonella isolates (recovered from retail raw chicken carcasses in China), harboring Class I integrin, presented a significantly $(p<0.05)$ higher resistance to tetracycline, ampicillin,

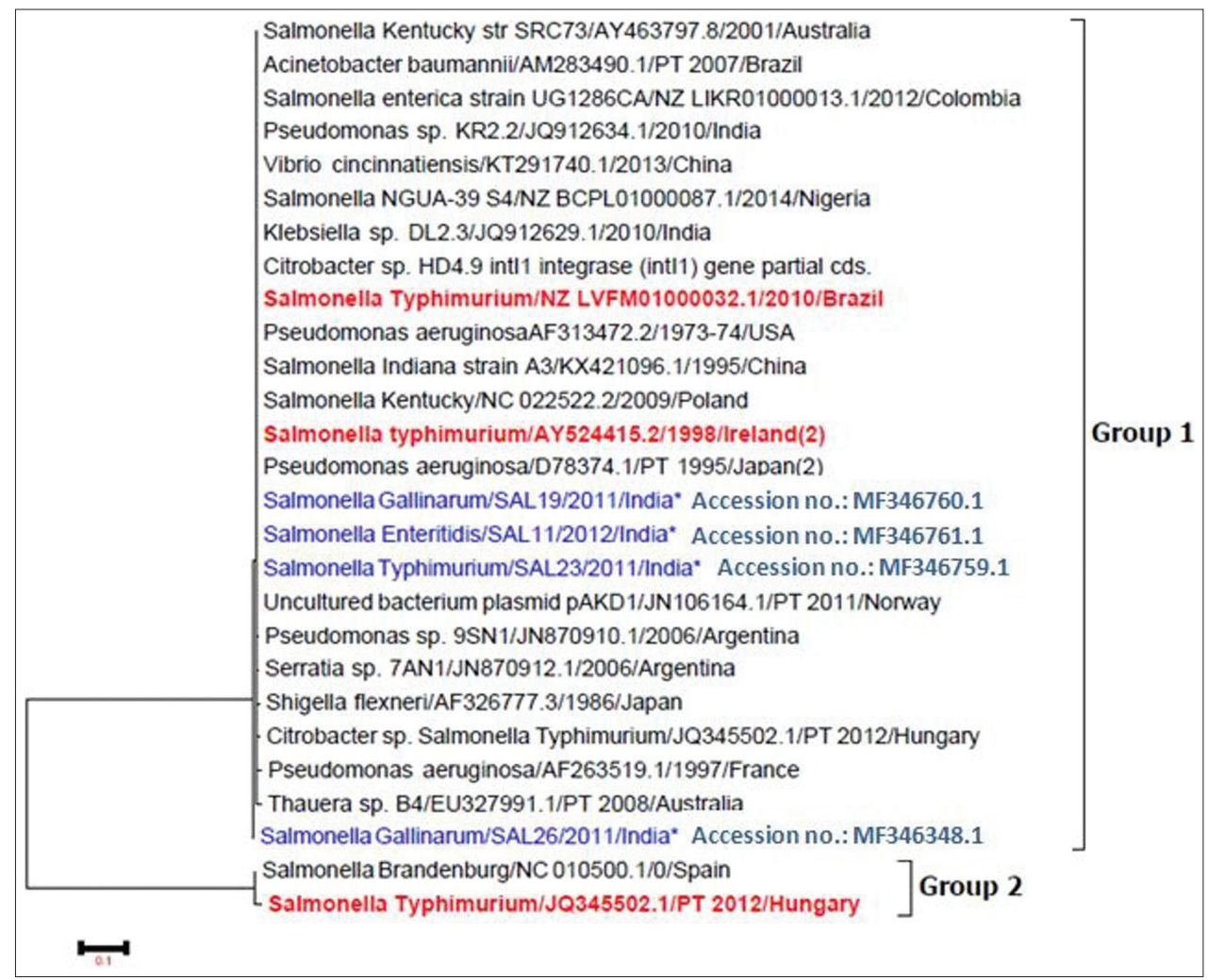

Figure-2: The evolutionary history of intI1 inferred using the neighbor-joining method. The evolutionary distances were computed using the maximum composite likelihood method. The analysis involved 27 nucleotide sequences. The tree with the highest log likelihood $(-879.2015)$ is shown. The tree is drawn to scale, with branch lengths measured in the number of substitutions per site.

Table-1: Number of Salmonella isolates with or without Class 1 integron in relation to their resistance toward different antimicrobials.

\begin{tabular}{|c|c|c|c|}
\hline Antibiotics & $\begin{array}{l}\text { Total number of } \\
\text { resistance isolates }\end{array}$ & $\begin{array}{l}\text { Class } 1 \text { Integron-positive } \\
\text { isolates }(n=40)(\%)\end{array}$ & $\begin{array}{c}\text { Class } 1 \text { Integron-negative } \\
\text { isolates }(n=3)(\%)\end{array}$ \\
\hline \multicolumn{4}{|l|}{$\beta$-lactams } \\
\hline Ampicillin & 2 & $2(5.0)$ & $0(-)$ \\
\hline Carbenicillin & 4 & $3(7.5)$ & $1(33.3)$ \\
\hline Cefotaxime & 3 & $3(7.5)$ & $0(-)$ \\
\hline \multicolumn{4}{|l|}{ Aminoglycosides } \\
\hline Gentamicin & 7 & $7(17.5)$ & $0(-)$ \\
\hline Kanamycin & 3 & $2(5.0)$ & $1(33.3)$ \\
\hline Streptomycin & 4 & $4(10.0)$ & $0(-)$ \\
\hline Spectinomycin & 10 & $10(25.0)$ & $0(-)$ \\
\hline \multicolumn{4}{|l|}{ Chloramphenicol } \\
\hline Chloramphenicol & 0 & $0(-)$ & $0(-)$ \\
\hline \multicolumn{4}{|l|}{ Tetracyclines } \\
\hline Tetracycline & 16 & $14(35.0)$ & $2(66.7)$ \\
\hline \multicolumn{4}{|l|}{ Sulfonamides } \\
\hline Co-trimoxazole & 13 & $12(30.0)$ & $1(33.3)$ \\
\hline Sulfafurazole & 27 & $25(62.5)$ & $2(66.7)$ \\
\hline \multicolumn{4}{|c|}{ Quinolones and fluoroquinolone } \\
\hline Ciprofloxacin & 39 & $37(92.5)$ & $2(66.7)$ \\
\hline Enrofloxacin & 32 & $30(75.0)$ & $2(66.7)$ \\
\hline Norfloxacin & 32 & $30(75.0)$ & $2(66.7)$ \\
\hline Nalidixic acid & 41 & $38(95.0)$ & $3(100)$ \\
\hline
\end{tabular}


Table-2: Number of isolates of different serotypes in relation to their carriage of Class 1 integrons.

\begin{tabular}{lccr}
\hline Serovar & Total number of isolates & \multicolumn{2}{c}{ Class 1 Integron-positive isolates } \\
\cline { 3 - 4 } & & $\boldsymbol{n}$ & \% (95\% CI) \\
\hline Salmonella Gallinarum & 34 & 31 & $91.18(76.3-98.0)$ \\
Salmonella Typhimurium & 5 & 5 & $100.00(47.8-100.0)$ \\
Salmonella Enteritidis & 2 & 2 & $100.00(15.8-100.0)$ \\
\hline
\end{tabular}

trimethoprim-sulfamethoxazole, amoxicillin-clavulanic acid, chloramphenicol, kanamycin, gentamicin, ceftiofur, cefoxitin, and amikacin compared with the average resistance rates [14]. The resistance of E. coli isolates toward sulfafurazole, trimethoprim, streptomycin, gentamicin, kanamycin, tobramycin, chloramphenicol, and amoxicillin was found to be associated with integron existence [15]. Similarly, another study has shown resistance against sulfonamides, trimethoprim, streptomycin, chloramphenicol, amoxicillin, tetracycline, and neomycin to be associated with integron existence [16]. Differences between intI1-positive and intI1-negative isolates in resistance to gentamicin, streptomycin, aminosidine, triple sulfonamides, and trimethoprim combined with sulfamethoxazole have been suggested to be significantly higher in intI1-positive compared with intI1-negative isolates [17]. This non-significant difference can be due to a lower number of isolates $(3 / 43)$ without integrons obtained in this study. In our study, 35/40 (87.5\%) intIl-positive isolates were MDR (resistant to $\geq 4$ antibiotics) which support the hypothesis of an association between the occurrence of Class 1 integrons and emerging MDR in Salmonella.

The outcome of phylogenetic analyses interprets the genetic similarity of Salmonella isolates of the current isolates to other isolates from different parts of the world, including India (Figure-2).

\section{Conclusion}

Based on our studies, we can conclude that the high prevalence of Class 1 integrons indicates the high potential of isolates for horizontal transmission of antimicrobial genes, especially among Gram-negative organisms. Further, elaborate study with more number of isolates would statistically improve our understanding about the role of this mechanism of resistance toward different antimicrobials, thus formulating targeted strategies for the amelioration of AMR.

\section{Authors' Contributions}

NKM and NJ diagnosed the disease collected samples. RG conceptualized and planned the study. RG, SLC, and SK carried out the laboratory work. VGJ carried out a phylogenetic analysis. RG analyzed the data. All authors read and approved the final manuscript.

\section{Acknowledgments}

The authors are thankful to Dean, College of Veterinary Sciences, Lala Lajpat Rai University of
Veterinary and Animal Sciences, India for providing necessary facilities and financial support for carrying out the research work.

\section{Competing Interests}

The authors declare that they have no competing interests.

\section{Publisher's Note}

Veterinary World remains neutral with regard to jurisdictional claims in published institutional affiliation.

\section{References}

1. Idrees, M., Mussarat, U., Badshah, Y., Qadir, M. and Bokhari, H. (2011) Prevalence of antimicrobial resistance and integrons in Escherichia coli from Punjab, Pakistan. Braz. J. Microbiol., 42(2): 462-466.

2. Cambray, G., Guerout, A.M. and Mazel D. (2010) Integrons. Annu. Rev. Genet., 44: 141-166.

3. Fluit, A.C. (2005) Towards more virulent and antibiotic-resistant Salmonella? FEMS Immunol. Med. Microbiol., 43(1): 1-11.

4. Li, B., Hu, Y., Wang, Q., Yi, Y., Woo, P.C.Y., Jing, H., Zhu, B. and Liu, C.H. (2013) Structural diversity of class 1 integrons and their associated gene cassettes in Klebsiella pneumonia isolates from a hospital in China. PLoS One, 8(9): e75805

5. Carattoli, A. (2003) Plasmid-mediated antimicrobial resistance in Salmonella enterica. Curr. Issues Mol. Biol., 5(4): 113-122.

6. Gupta, R., Jindal, N., Arora, D., Singh, M. and Kapoor, P.K. (2016) Detection of Salmonella Typhimurium, Salmonella Enteritidis and Salmonella Gallinarum from suspected cases of fowl typhoid in poultry in Haryana. Indian Vet. J., 93(7): 44-47.

7. Oosterik, L.H., Peeters, L., Muruku, I., Goddeeris, B.M. and Butaye, P. (2014) Susceptibility of avian pathogenic Escherichia coli from laying hens in Belgium to antibiotics and disinfectants and integron prevalence. Avian Dis., 58(2): 271-278.

8. Tamura, K., Stecher, G., Peterson, D., Filipski, A. and Kumar, S. (2013) MEGA6: Molecular evolutionary genetics analysis version 6.0. Mol. Biol. Evol., 30(12): 2725-2729.

9. Lu, Y., Zhao, H., Sun, J., Liu, Y. and Zhou, X. (2014) Characterization of multidrug-resistant Salmonella enterica serovars Indiana and Enteritidis from chickens in eastern China. PLoS One, 9(5): e96050.

10. Kwon, H.J., Kim, T.E., Cho, S.H., Seol, J.G. and Kim, B.J. (2002) Distribution and characterization of class 1 integrons in Salmonella enterica serotype Gallinarum biotype gallinarum. Vet. Microbiol., 89(4): 303-309.

11. Trongjit, S., Angkititrakul, S., Tuttle, R.E., Poungseree, J., Padungtod, P. and Chuanchuen, R. (2017) Prevalence and antimicrobial resistance in Salmonella enterica isolated from broiler chickens, pigs and meat products in ThailandCambodia border provinces. Microbiol. Immunol., 61(1): 23-33.

12. Vo, A.T., Duijkeren, E.V., Gaastra, W. and Fluit, A.C. (2010) 
Antimicrobial resistance, class 1 integrons, and genomic island 1 in Salmonella isolates from Vietnam. PLoS One, 5(2): e9440.

13. Firoozeh, F., Zahraei-Salehi, T., Shahcheraghi, F., Karimi, V. and Aslani, M.M. (2012) Characterization of class I integrons among Salmonella enterica serovar Enteritidis isolated from humans and poultry. FEMS Immunol. Med. Microbiol., 64(2): 237-243.

14. Meng, X., Zhang, Z., Li, K., Wang, Y., Xia, X., Wang, X., Xi, M., Meng, J., Cui, S. and Yang, B. (2017) Antibiotic susceptibility and molecular screening of class I integron in Salmonella isolates recovered from retail raw chicken carcasses in China. Microb. Drug Resist., 23(2): 230-235.
15. White, P.A., Mciver, C.J. and Rawlinson, W.D. (2001) Integrons and gene cassettes in the Enterobacteriaceae. Antimicrob. Agents Chemother., 45(9): 2658-2661.

16. Marchant, M., Vinue, L., Torres, C. and Moreno, M.A. (2013) Change of integrons over time in Escherichia coli isolates recovered from healthy pigs and chickens. Vet. Microbiol., 163(1-2): 124-132.

17. Piccirillo, A., Giovanardi, G., Dotto, G., Grilli, C., Montesissa, C., Boldrin, C., Salata, C. and Giacomelli, M. (2014) Antimicrobial resistance and class 1 and 2 integrons in Escherichia coli from meat Turkeys in Northern Italy. Avian Pathol., 43(5): 396-405.

$* * * * * * * *$ 